\title{
Remapping and mutation analysis of benign adult familial myoclonic epilepsy in a Japanese pedigree
}

\begin{abstract}
Satsuki Mori ${ }^{1,5}$, Masayuki Nakamura ${ }^{1,5}$, Takeshi Yasuda ${ }^{2}$, Shu-ichi Ueno ${ }^{3}$, Sunao Kaneko ${ }^{4}$ and Akira Sano ${ }^{1}$
Benign adult familial myoclonic epilepsy (BAFME), alternatively named familial adult myoclonic epilepsy $1 /$ familial cortical myoclonic tremor with epilepsy 1 (FAME1/FCMTE1), is a hereditary epileptic syndrome characterized by autosomal dominant inheritance, adult-onset tremulous hand movement, myoclonus, infrequent epileptic seizure and non-progressive course without cerebellar ataxia and dementia. We previously reported evidence for linkage of BAFME to the region between D8S1784 and D8S1694 on chromosome 8q. Subsequently, other research groups reported mapping of the same clinical syndrome to different chromosomal loci, $2 p$ and 5p, in Italian (FAME2/FCMTE2) and French (FAME3/FCMTE3) families, respectively. In this study, we performed a genome-wide linkage analysis using $10 \mathrm{~K}$ single-nucleotide polymorphism arrays and additional microsatellite markers to reconfirm the BAFME-linked region. The BAFME-linked region was mapped to $7.16 \mathrm{Mb}$ spanned by rs 1898287 and rs2891799 on chromosomes 8q23.3-8q24.13 with a maximum two-point logarithm of odds score of 6.0 for the marker rs 1021897 . Sequence analysis and copy-number variant analysis of all 38 genes localized in the candidate region were performed, but no pathogenic mutation was identified. We conclude that the etiology of BAFME remains to be solved, and further genetic studies, which may require analysis in non-coding regions of a gene, introns or intergenic spacer regions, are necessary to reveal its unknown mutations.
\end{abstract}

Journal of Human Genetics (2011) 56, 742-747; doi:10.1038/jhg.2011.93; published online 18 August 2011

Keywords: array-based comparative genomic hybridization; FCMTE; genome-sequencing; genome-wide scan; SNP analysis

\section{INTRODUCTION}

Yasuda proposed the term 'benign adult familial myoclonic epilepsy (BAFME)' in $1991,{ }^{1}$ which was characterized by the following features: (1) autosomal dominant inheritance, tremulous finger movement and/or myoclonus of the extremities after adolescence; (2) infrequent epileptic seizure; (3) abnormality of polyspikes and waves on examination by electroencephalogram (EEG) and marked photosensitivity; (4) enlarged cortical components of somatosensory-evoked potential (SEP); (5) enhanced long-loop reflex (C-reflex); (6) positive spikes preceding myoclonus ascertained with the jerk-locked averaging method; and (7) benign non-progressive course without cerebellar ataxia and dementia. ${ }^{1,2}$

Historically, various names have been given to the syndrome that show cortical myoclonic tremor and epilepsy: hereditary tremor with epileptiform seizures, ${ }^{3}$ heredofamilial tremor and epilepsy, ${ }^{4}$ cortical tremor, ${ }^{5}$ familial essential myoclonus and epilepsy, ${ }^{6}$ BAFME, ${ }^{1}$ familial adult myoclonic epilepsy (FAME) ${ }^{7}$ familial benign myoclonus epilepsy of adult onset, ${ }^{8}$ familial cortical tremor with epilepsy, ${ }^{9}$ autosomal dominant cortical myoclonus and epilepsy ${ }^{10}$ and familial cortical myoclonic tremor with epilepsy (FCMTE). ${ }^{11}$
Previous genetic studies, including our earlier report, ${ }^{2}$ identified three different chromosomal loci, 8q $\mathrm{q}^{2,12}$ (BAFME, FCMTE1), 2p $\mathrm{p}^{10,13,14}$ (FCMTE2) and $5 \mathrm{p}^{15}$ (FCMTE3) in Japanese, Italian and French pedigrees, respectively. The European patients seemed to show a more progressive disease course than Japanese patients. ${ }^{11}$ Compared with Japanese patients, the European patients also appear to be less susceptible to the effects of antiepileptic drugs. Worsening of epilepsy over the years leading to symptoms such as 'inability to use hands or legs' has been reported, especially in the European pedigrees. ${ }^{16,17,18}$ In addition, cognitive decline and mental retardation were also reported only in the European pedigrees. ${ }^{10,16,18}$ The causative genes of FCMTE2 and FCMTE3 have not yet been identified.

In this study, to reconfirm and perform fine mapping of the BAFME (FCMTE1) loci, we performed a genome-wide linkage analysis using $10 \mathrm{~K}$ single-nucleotide polymorphism (SNP) arrays and additional microsatellite markers. Furthermore, to reveal the genetic cause of BAFME, mutation analyses were performed in all 38 genes localized in the candidate region, including non-protein-coding mRNA genes as reported in the human genome database constructed by NCBI (Genome Reference Consortium Human Build 37.1).

${ }^{1}$ Department of Psychiatry, Kagoshima University Graduate School of Medical and Dental Sciences, Kagoshima, Japan; ${ }^{2}$ Kurashikikinen Hospital, Okayama, Japan; ${ }^{3}$ Department of Neuropsychiatry, Neuroscience, Ehime University Graduate School of Medicine, Ehime, Japan and ${ }^{4}$ Department of Neuropsychiatry, Hirosaki University Graduate School of Medical, Hirosaki, Japan

5These authors contributed equally to this work and they are co-first authors.

Correspondence: Professor A Sano, Department of Psychiatry, Kagoshima University Graduate School of Medical and Dental Sciences, 8-35-1 Sakuragaoka, Kagoshima 890-8520, Japan.

E-mail: sano@m3.kufm.kagoshima-u.ac.jp

Received 25 April 2011; revised 11 July 2011; accepted 15 July 2011; published online 18 August 2011 


\section{MATERIALS AND METHODS}

\section{Patients}

Linkage analysis was performed in a large Japanese family whose clinical phenotypic features were already described by Yasuda ${ }^{1}$ and Mikami et al. ${ }^{2}$ Participants' genomic DNA was extracted from peripheral leukocytes using standard methods after informed consent and approval by the local ethics committee was obtained.

Yasuda ${ }^{1}$ reported detailed clinical features and electrophysiological findings of II-4, II-5, II-7, II-10, II-15, II-19, III-3, III-4, III-7, III-8 and III-14. The amplitude of the P25 and N33 components of SEPs was increased $(20-55 \mu \mathrm{V})$ by stimulation of the median nerve at the wrist. Following SEPs, an abrupt increase in the muscle discharge was induced with a latency of $38-45 \mathrm{~ms}$ at rest from the ipsilateral thenar muscle by stimulation at the same site (C-reflex). All of them showed polyspikes and wave complex or spikes and wave complex in the EEG. The detailed characteristics in II-6, III-10, III-12, III-13 and III-15 were not described in the paper. However, all of them showed tremulous finger movements, enlarged cortical components of SEPs, C-reflex and abnormal EEG. We diagnosed III-2, III- 6 and III-11 as unaffected because all of them did not show giant-SEPs, C-reflex and abnormal EEG. We also diagnosed II-2 and
II-14 as unaffected because they have never showed any clinical signs of BAFME even at an age of over 80 years.

The clinical diagnosis of II-18 was altered to 'unaffected' from 'affected'. II-18 (Figure 1) was diagnosed with BAFME in our previous study as reported by Mikami et al., ${ }^{2}$ because II-18 showed slight tremulous finger movement and increased muscle discharge following electric stimulation of the median nerve at the wrist. However, in this study we reconsidered the diagnosis of II-18 as unaffected because of the following five reasons: (I) II-18 took theophylline for the treatment of asthma, which was probably responsible for the tremulous hand movement. (II) The amplitude of the P25 and N33 components of SEP of II-18 was less increased $(11 \mu \mathrm{V})$ than the patients of this family $(20-55 \mu \mathrm{V})$ by stimulation of the median nerve at the wrist. (III) Usually, C-reflex was induced from the ipsilateral thenar muscle by stimulation at the same site; however, an increased muscle discharge in II-18 was induced only from the contralateral thenar muscle. (IV) Subsequently paroxysmal discharges have not been observed in the electroencephalogram (EEG). (V) II-18 had never experienced epileptic seizures even over the age of 70 years.

Altogether, the 27 available family members, including 16 affected patients and 11 unaffected individuals, were studied (Figure 1).

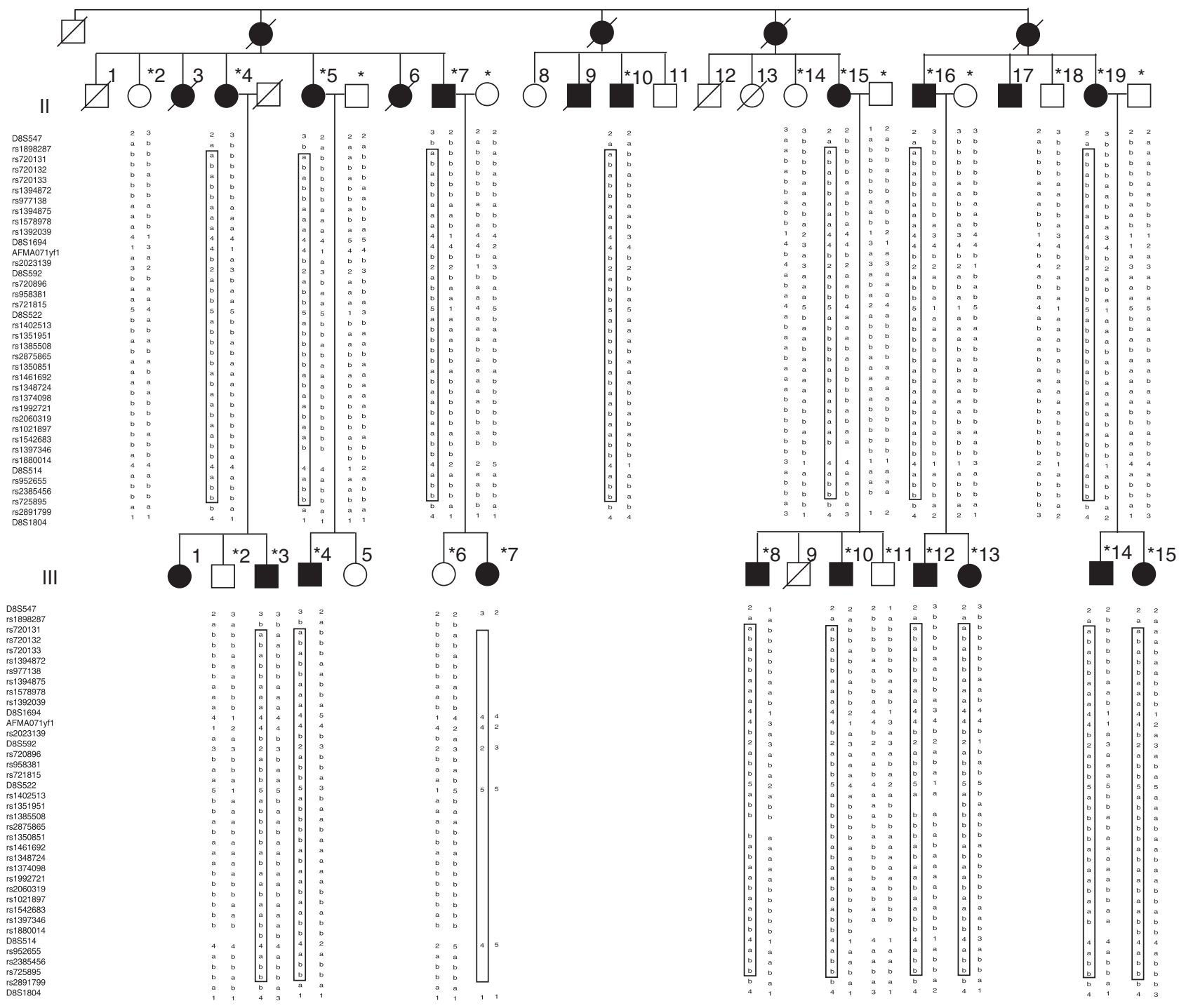

Figure 1 Pedigree and haplotypes of a Japanese family with benign adult familial myoclonic epilepsy (BAFME). Affected status is indicated by a filled square (male) or circle (female). Asterisks indicate individuals who were sampled and analyzed. The haplotypes are shown under each individual, with the disease haplotype boxed. An empty space in the haplotype indicates that the individual was not genotyped at that microsatellite marker or single-nucleotide polymorphism locus. 


\section{Linkage and haplotype analyses}

Genome-wide linkage analysis was performed on 15 affected patients and 11 unaffected individuals (Figure 1) using the GeneChip Human Mapping 10K 2.0 Xba Array (Affymetrix, Santa Clara, CA, USA). Although there were 27 DNA samples from family members, one sample from the patient III-7 (Figure 1) was excluded owing to the bad quality of the DNA sample submitted for the array analysis. All experimental procedures were according to the Affymetrix protocol. Genotyping of SNPs was performed with the GeneChip DNA Analysis Software 2.0 (Affymetrix).

After genotyping, linkage analysis was performed using GeneSpring GT2.0 (autosomal dominant model with $100 \%$ penetrance, disease allele frequency of 0.001 , recombination fractions $(\theta)$ of $0.00,0.10,0.20,0.30$ and 0.40 ) (Agilent Technologies, Santa Clara, CA, USA).

Genetic evidence for recombination in BAFME was based on haplotype analysis. SNP analysis and additional microsatellite markers analysis were performed in the 27 available family members. Genotyping seven microsatellite markers on chromosomes 8q23.3-24.13: D8S547, D8S1694, AFMA071yf1, D8S592, D8S522, D8S514 and D8S1804 (Figure 1) were determined with amplified alleles, which were visualized as bands on $6 \%$ denaturing polyacrylamide gels.

\section{DNA sequence analysis using direct sequencing}

The interval of $7.16 \mathrm{Mb}$ between rs1898287 and rs2891799 includes 38 genes reported in the NCBI database (Figure 3). We sequenced all 38 genes, including non-protein-coding RNA genes in at least one affected individual (Figure 1: II-4 or II-7). In all genes, polymerase chain reaction primers (sequences available upon request) were designed to amplify all coding exons (non-coding RNA exons), $5^{\prime}$ untranslated regions, predicted promoter regions (approximately 730-1130 bp upstream of each $5^{\prime}$ gene flanking region) and intronic regions surrounding each exon. Amplified fragments were directly sequenced using a BigDye Terminator v.3.1 Cycle sequencing system on an automated ABI PRISM3100-Avant Genetic Analyzer (Applied Biosystems, Foster City, CA, USA). SNPs were assessed using the NCBI SNP database. In some cases, additional sequence analyses were performed in the 27 available family members to confirm whether a given nucleotide variant resulted in benign polymorphisms or was disease causing.

\section{DNA sequence analysis using genome sequencing}

To establish high confidence in the sequencing, an $8.4 \mathrm{Mb}$ interval spanned by D8S547 and D8S1804, which included the linked region spanned by rs 1898287 and rs2891799, was sequenced using genome sequencing in one affected individual (Figure 1: II-4). Briefly, targeted enrichment of the region between markers D8S547 and D8S1804 was performed by hybridization to NimbleGen Sequence Capture 2.1M programmable microarrays (Roche NimbleGen, Madison, WI, USA) and then each enriched shotgun genomic library was sequenced on a Roche Genome Sequencer FLX System (Roche Diagnostics, Indianapolis, IN, USA). Because highly similar sequences in the genome are not identified easily with current sequencing platforms, approximately $18.2 \%$ of the primary target region was lost before probe design to avoid capture of homologous repetitive elements. We captured and sequenced the target region to a mean coverage of $\times 20$. We determined bases from the primary sequencing data, using the standard GS FLX analysis software (Roche Diagnostics). Its accuracy in assembling 500-base reads was approximately $96.77 \%$. All experimental procedures were according to the Roche protocol.

\section{Copy-number variants analysis using array-based comparative genomic hybridization}

Whole genome copy-number variant $(\mathrm{CNV})$ analysis was performed in one affected patient (Figure 1: II-4) using the Genome-Wide Human SNP Array 6.0 (Affymetrix), which features 1.8 million genetic markers, including more than 906600 SNPs and more than 946000 comparative genomic hybridization probes. Labeling and hybridization were performed according to the Affymetrix protocol. Raw data were acquired from the scanner and transferred to a Genotyping Console 3.0.2 (Affymetrix) to determine the genotypes and CNVs from fluorescence intensity data.

In all of the exons of each of the 38 genes localized in the candidate region, signal intensities for $\mathrm{CN}$ predictions of both duplications and deletions were investigated in detail from the raw data. In the potentially duplicated region, real-time polymerase chain reaction assays using TaqMan Copy Number Assays (Applied Biosystems) and a 7300 Real Time PCR System (Applied Biosystems) were designed.

\section{RESULTS}

\section{Genome-wide linkage and haplotype analyses}

We performed genome-wide scan using the DNA arrays to reconfirm the evidence for linkage of BAFME. A maximum two-point logarithm of odds (LOD) score of $6.0(\theta=0)$ was obtained for marker rs1021897 on chromosome 8q24.12 (Figure 2). In addition, two-point LOD scores higher than $3.0(\theta=0)$ were also obtained with markers rs721815 and rs1350851 on chromosome 8q24.12.

Haplotype analysis using SNPs and additional microsatellite markers analysis revealed a common haplotype delimited by rs720131 and rs725895 on chromosome 8q24.11-8q24.13 segregated in all the available patients (Figure 1). The proximal recombination site is between rs1898287 and rs720131 and the distal site is between rs725895 and rs2891799.

These results demonstrate the linked region of BAFME to be within the approximately $7.16 \mathrm{Mb}$ spanned by rs1898287 and rs2891799 on chromosomes 8q23.3-8q24.13 (Figure 1).

\section{DNA sequence analysis}

The candidate region contained 38 genes. None of the genes in the candidate region showed any apparent relationship to chromosome 2 or chromosome 5 . We sequenced all 38 genes by direct sequencing and genome sequencing to establish a high level of confidence in the sequences. The direct sequencing was performed in all coding exons, 5 -untranslated regions, predicted promoter regions and introns surrounding each exon. In the direct sequencing, we detected 30 nucleotide variants, which had not been recorded in the NCBI SNP database (Figure 3), although 25 of these were also observed in normal control samples. The other five intronic heterozygous nucleotide alterations were as follows: the c.1471-3A $>\mathrm{G}$ substitution in the RAD21 gene, the c. $-158 \mathrm{G}>\mathrm{C}$ substitution in the SAMD12 gene, the c.293-44T $>$ A substitution in the COLEC10 gene, the c.307-15A $>$ G substitution in the MRPL13 gene and the c.1746+5G > A substitution

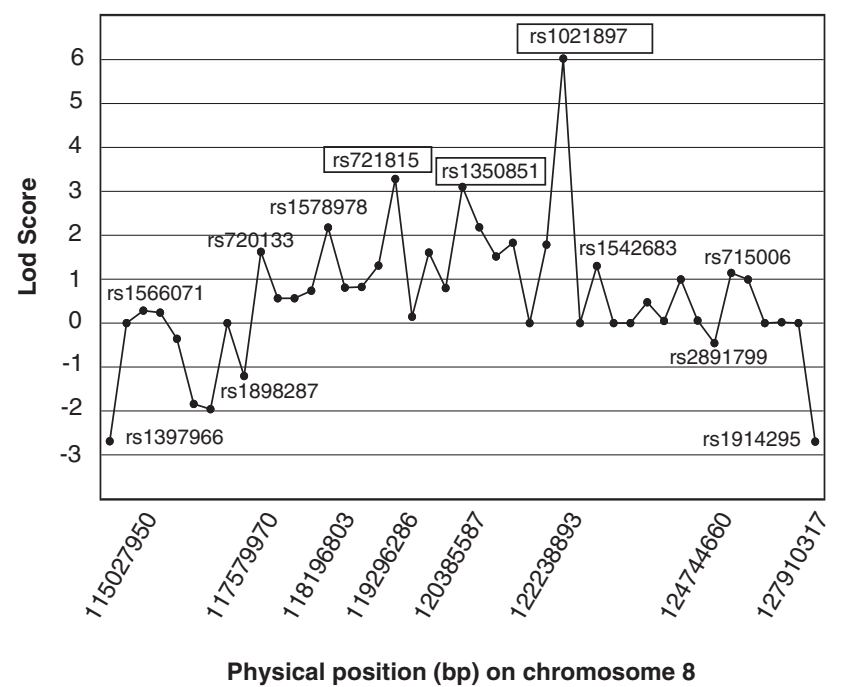

Figure 2 Two-point logarithm of odds (LOD) scores for genetic location on chromosome 8q23.3-8q24.21. Two-point LOD scores higher than 3.0 are boxed. A maximum two-point LOD score of 6.0 for marker rs 1021897 was obtained. 


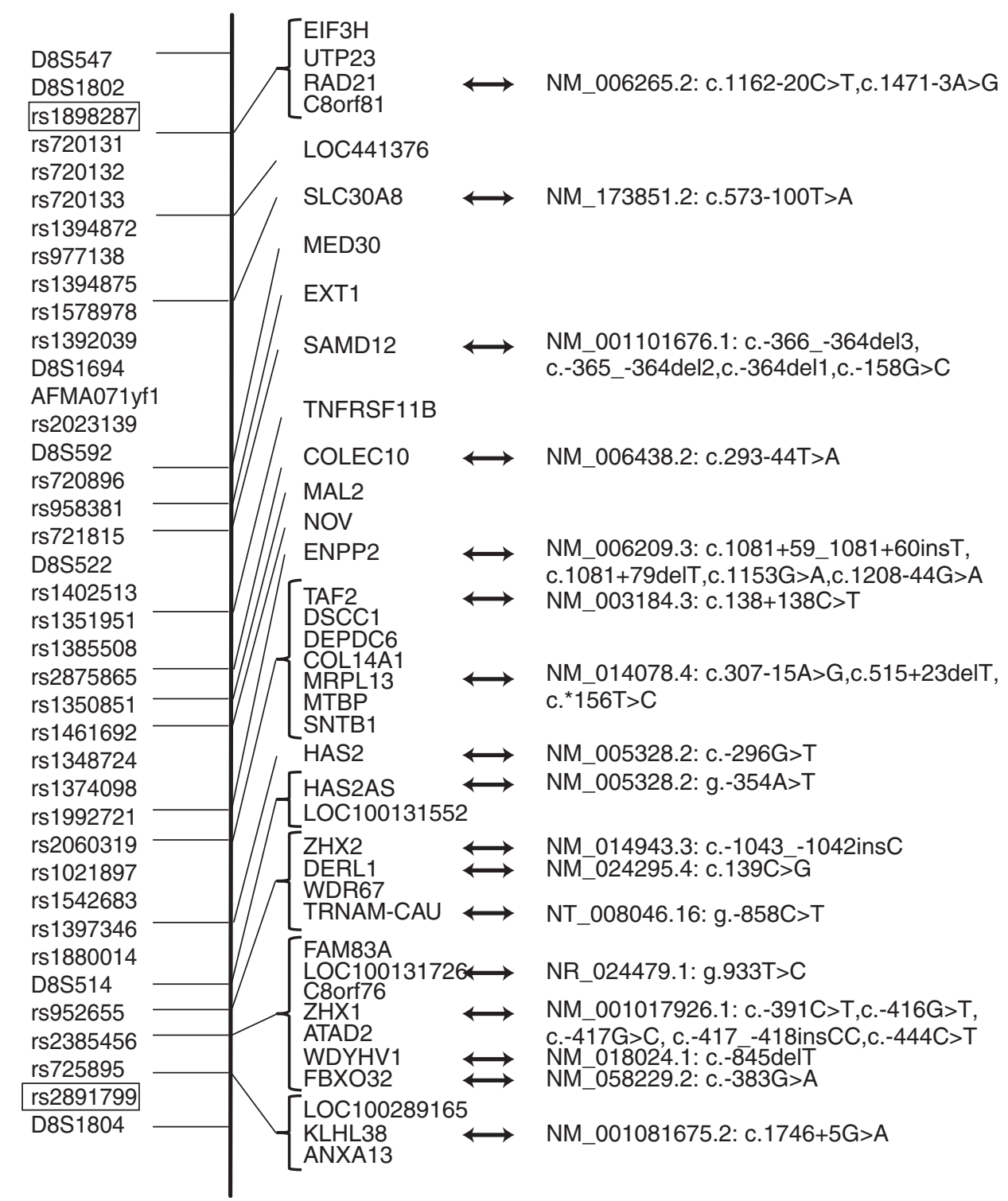

Figure 3 The 38 genes in the benign adult familial myoclonic epilepsy (BAFME) candidate region and the novel 30 nucleotide variants identified in this study. The 30 nucleotide variants had not been recorded by the NCBI SNP database; 25 of which were also observed in normal control samples. The interval between rs1898287 (boxed) and rs2891799 (boxed) indicates the linked region of BAFME. Double-headed arrows indicate the gene and the novel nucleotide variants in it.

in the KLHL13 gene. However, an apparent causative gene for BAFME was not detected.

On the other hand, 17-nucleotide changes in the coding region sequence and 2240-nucleotide changes in the non-coding region sequence, which had not been recorded by the NCBI SNP database, were detected by genome sequencing. The 17-nucleotide changes in the coding region sequence were confirmed by direct sequencing again. However, direct sequencing revealed that none of these 17-nucleotide changes in the coding region sequence actually existed in the patient.

\section{CNV analysis}

$\mathrm{CNV}$ analysis using array-based comparative genomic hybridization was performed to detect the mutations, which could not be found by sequence analysis. In all of the exons of the 38 genes localized in the candidate region, signal intensities for $\mathrm{CN}$ predictions of both duplications and deletions were investigated in detail from the raw data. In the potentially duplicated region, additional $\mathrm{CNV}$ analysis using TaqMan Copy Number Assays was then performed on exons 3-5 of the NOV gene, exon 3 of the ENPP2 gene, exon 7 of the MRPL13 gene and exons 41 and 42 of the COL14A1 gene in II-4, II-5, II-19, III-3, III-4 (Figure 1) and the control sample. However, no CNV mutation was detected.

\section{DISCUSSION}

In this study, the clinical diagnosis of II-18 was reconsidered and altered to 'unaffected' from 'affected'. Slight tremulous hand movement, which II-18 showed, was deemed a side effect of theophylline, which the subject had been treated with. In addition, II-18 did not show enough electrophysiological features of cortical reflex myoclonus (giant-SEPs, C-reflexes) as well as no paroxysmal discharges in the EEG, although all other patients in the pedigree showed these features. 


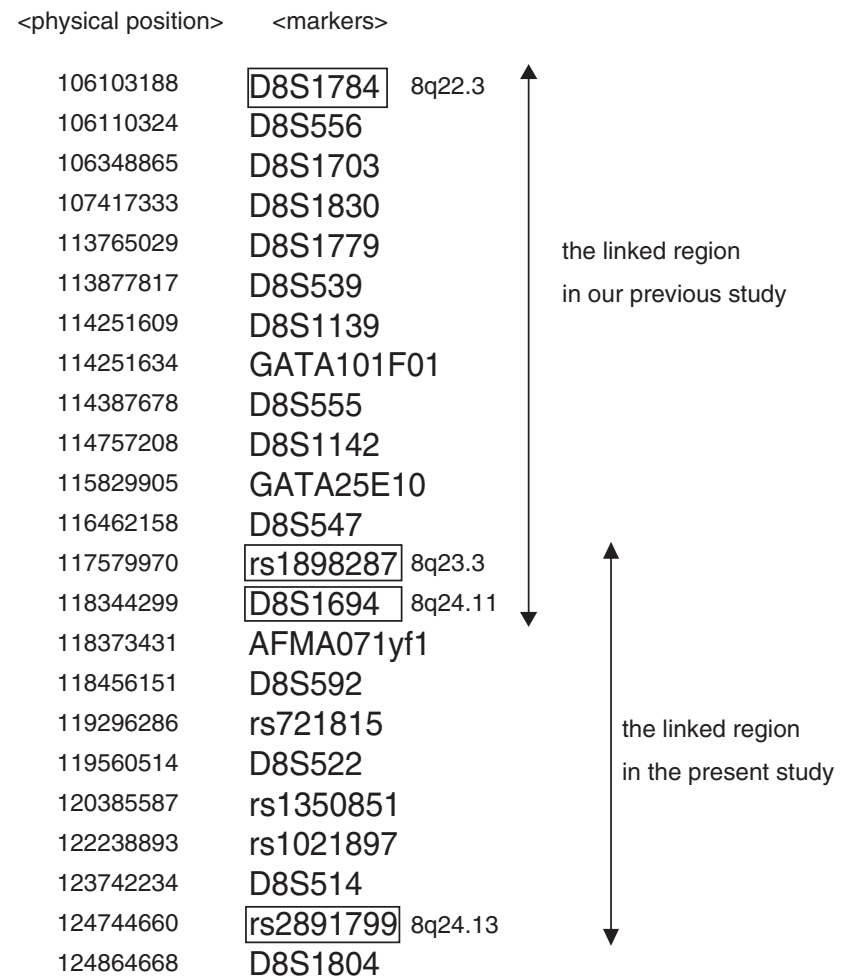

Figure 4 Remapped region of benign adult familial myoclonic epilepsy (BAFME). Upper double-headed arrow indicates the BAFME linked region in our previous study. Inferior double-headed arrow indicates the linked region in this study.

Van Rootselaar et al. ${ }^{11}$ elucidated the diagnostic criteria of FCMTE. According to their diagnostic criteria, a member with myoclonic tremor, which is not accompanied by EEG changes and electrophysiological features of cortical reflex myoclonus (giant-SEPs, C-reflexes), is considered as probably affected, if at least one member of the family have cortical myoclonic tremor and at least one have epileptic attacks. However, they emphasized that in cases with possible side effects of medication, like the situation of II-18, electrophysiological data are required to determine whether the tremor stems from cortical hyperexcitability.

Linkage analysis was performed in the same large Japanese family as our previous report by Mikami et al. ${ }^{2}$ In this study, we reconfirmed positive linkage for $8 \mathrm{q}$ by a genome-wide linkage analysis using SNP arrays and additional microsatellite markers. Genome-wide linkage study revealed negative linkage for FAME2/FCMTE2 (2p11.1-q12.2) and FAME3/FCMTE3 (5p15.31-p15) loci. Compared with our previous study, the linkage evidence was shifted from an interval between D8S1784 and D8S1694 (8q22.3-q24.11) to an interval between rs1898287 and rs2891799 (8q23.3-q24.13) with a slight overlap (Figure 4). The slight telomeric shift of the linked region is due to the alterations in the clinical diagnosis of II-18 and changes in the physical positions of microsatellite markers revealed by data updates. Investigation of another family with clear diagnostic conditions is required to narrow the linkage region.

Although mutational analysis of all 38 genes did not reveal a distinctive causative mutation or changes in copy number in the candidate region, 2240-nucleotide changes in the non-coding region sequence, which had not been recorded by the NCBI SNP database, were detected by genome sequencing. It is possible that causative mutations of BAFME exist in the 2240-nucleotide changes. There may be as yet unidentified genes including the non-protein-coding transcript that have structural, regulatory or other unknown functions. ${ }^{19,20}$ However, it is difficult to identify a functional effect deriving from any of the 2240-nucleotide changes in non-coding regions. Further investigations such as RNA expression studies analyzing transcripts from post-mortem brains from affected patients are required to confirm the potential unknown effects of the non-coding mutation(s).

Gene inversion is another possible cause of BAFME, because gene inversion encompassing one whole exon or several whole exons cannot be excluded by CNV analysis or sequence analysis. Further analysis, such as fluorescent in situ hybridization, is required to detect such aberrations in chromosomal structure.

Although there may be some degree of unclarity of the clinical diagnosis of II-18, which was responsible for another possible reason as to why we could not find the responsible genes, the present investigated family of BAFME was regarded as a core family of BAFME among various families with clearer diagnostic conditions. However, in future, it could be necessary to investigate another family with further clearer diagnostic conditions of BAFME.

Most idiopathic epilepsies are caused by mutations in ion channels or neuroreceptor components, ${ }^{21-23}$ but no gene in the linked region of BAFME is known to encode any ion channel or neuroreceptor component. The proteins known to be related to epilepsy function in CNS development, ${ }^{24}$ synaptic strength at the excitatory synapses, ${ }^{25}$ release of neurotransmitters, ${ }^{26}$ regulation of glycogen metabolism ${ }^{27}$ or calcium homeostasis, ${ }^{28}$ and so on. In the linked region, there is no gene encoding a protein that has such functions. A novel epileptogenic mechanism may therefore underlie BAFME.

In conclusion, we detected strong linkage evidence of BAFME (FAME1/FCMTE1) to chromosome 8q23.3-24.13 again and remapped the BAFME locus by performing a genome-wide linkage study and including additional microsatellite markers. However, comprehensive mutation analyses of all 38 genes in the candidate region revealed no causative mutation. These results suggest that causative mutations of BAFME may exist in the non-coding region, which may include unidentified genes. In addition, it is possible that there are novel pathogenic mechanisms contributing to BAFME. Further investigations are necessary to detect the causative gene and mechanisms contributing to BAFME.

\section{ACKNOWLEDGEMENTS}

We thank all patients and their family members for their willingness to participate in this study. We also thank Mrs M. Ishigami, Mrs M. Imamura, Mrs H. Hiwatashi and Mrs A. Agemura for their excellent technical assistance. This work was supported in part by Grants-in-Aid for Research from the Ministry of Education, Culture, Sports, Science and Technology, Japan.

1 Yasuda, T. Benign adult familial myoclonic epilepsy (BAFME). Kawasaki Med. J. 17, 1-13 (1991).

2 Mikami, M., Yasuda, T., Terao, A., Nakamura, M., Ueno, S., Tanabe, H. et al. Localization of a gene for benign adult familial myoclonic epilepsy to chromosome 8q23.3-q24.1. Am. J. Hum. Genet. 65, 745-751 (1999).

3 Inoue, S. one pedigree of hereditary tremor with epileptiform seizures. Seishin Shink. Zasshi 53, 33-37 (1951).

4 Kudo, J., Kudo, T. \& Yamauchi, T. Seven families with heredofamilial tremor and epilepsy. Rinsho Shink. 24, 1-8 (1984).

5 Ikeda, A., Kakigi, R., Funai, N., Neshige, R., Kuroda, Y. \& Shibasaki, H. Cortical tremor: a variant of cortical reflex myoclonus. Neurology 40, 1561-1565 (1990).

6 Inazuki, G., Naito, H., Ohama, E., Kawase, Y., Honma, Y., Tokiguchi, S. et al. A clinical study and neuropathological findings of a familial disease with myoclonus and epilepsy - the nosological place of familial essential myoclonus and epilepsy (FEME). Seishin Shink. Zasshi 92, 1-21 (1990). 
7 Uyama, E., Tokunaga, M., Murakami, T., Kuwano, A., Kondo, I. \& Uchino, M. Familial adult myoclonus epilepsy: a new phenotype of autosomal dominant myoclonic epilepsy [abstract]. Ann. Neurol. 40, 505 (1996).

8 Okino, S. Familial benign myoclonus epilepsy of adult onset: a previously unrecognized myoclonic disorder. J. Neurol. Sci. 145, 113-118 (1997).

9 Okuma, Y., Shimo, Y. \& Shimura, H. Familial cortical tremor with epilepsy: an underrecognized familial tremor. Clin. Neurol. Neurosurg. 100, 75-78 (1998).

10 Guerrini, R., Bonanni, P., Patrignani, A., Brown, P., Parmeggiani, L. \& Grosse, P. Autosomal dominant cortical myoclonus and epilepsy (ADCME) with complex partial and generalized seizures: a newly recognized epilepsy syndrome with linkage to chromosome 2p11.1-q12.2. Brain 124, 2459-2475 (2001).

11 van Rootselaar, A. F., van Schaik, I. N., van den Maagdenberg, A. M., Koelman, J. H., Callenbach, P. M. \& Tijssen, M. A. Familial cortical myoclonic tremor with epilepsy: a single syndromic classification for a group of pedigrees bearing common features. Mov. Disord. 20, 665-673 (2005).

12 Plaster, N. M., Uyama, E., Uchino, M., Ikeda, T., Flanigan, K. M., Kondo, I. et al. Genetic localization of the familial adult myoclonic epilepsy (FAME) gene to chromosome 8q24. Neurology 53, 1180-1183 (1999).

13 de Falco, F. A., Striano, P., de Falco, A., Striano, S., Santangelo, R., Perretti, A. et al. Benign adult familial myoclonic epilepsy: genetic heterogeneity and allelism with ADCME. Neurology 60, 1381-1385 (2003).

14 Striano, P., Zara, F. \& Striano, S. Autosomal dominant cortical tremor, myoclonus and epilepsy: many syndromes, one phenotype. Acta Neurol. Scand. 111, 211-217 (2005).

15 Depienne, C., Magnin, E., Bouteiller, D., Stevanin, G., Saint-Martin, C., Vidailhet, M. et al. Familial cortical myoclonic tremor with epilepsy: the third locus (FCMTE3) maps to 5p. Neurology 74, 2000-2003.

16 van Rootselaar, A. F., Callenbach, P. M., Hottenga, J. J., Vermeulen, F. L., Speelman, H. D., Brouwer, O. F. et al. A Dutch family with 'familialcortical tremor with epilepsy'. Clinical characteristics and exclusion of linkage to chromosome 8q23.3-q24.1. J. Neurol. 249, 829-834 (2002).
17 Labauge, P., Amer, L. O., Simonetta-Moreau, M., Attane, F., Tannier, C., Clanet, M. et al. Absence of linkage to $8 \mathrm{q} 24$ in a European family with familial adult myoclonic epilepsy (FAME). Neurology 58, 941-944 (2002).

18 Elia, M., Musumeci, S. A., Ferri, R., Scuderi, C., Del Gracco, S., Bottitta, M. et al. Familial cortical tremor, epilepsy, and mental retardation: a distinct clinical entity? Arch. Neurol. 55, 1569-1573 (1998).

19 Amaral, P. P., Dinger, M. E., Mercer, T. R. \& Mattick, J. S. The eukaryotic genome as an RNA machine. Science 319, 1787-1789 (2008).

20 Amaral, P. P., Clark, M. B., Gascoigne, D. K., Dinger, M. E. \& Mattick, J. S. IncRNAdb: a reference database for long noncoding RNAs. Nucleic Acids Res. 39, D146-D151 (2011).

21 Avanzini, G., Franceschetti, S. \& Mantegazza, M. Epileptogenic channelopathies: experimental models of human pathologies. Epilepsia 48, 51-64 (2007).

22 Greenberg, D. A. \& Pal, D. K. The state of the art in the genetic analysis of the epilepsies. Curr. Neurol. Neurosci. Rep. 7, 320-328 (2007).

23 Christopher, A. R., Samuel, F. B. \& Steven, P. Mechanisms of human inherited epilepsies. Prog. Neurobiol. 87, 41-57 (2009).

24 Stromme, P., Mangelsdorf, M. E., Shaw, M. A., Lower, K. M., Lewis, S. M., Bruyere, H. et al. Mutations in the human ortholog of Aristaless cause X-linked mental retardation and epilepsy. Nat. Genet. 30, 441-445 (2002).

25 Kalachikov, S., Evgrafov, O., Ross, B., Winawer, M., Barker-Cummings, C., Martinelli Boneschi, F. et al. Mutations in LGI 1 cause autosomal-dominant partial epilepsy with auditory features. Nat. Genet. 30, 335-341 (2002).

26 Saitsu, H., Kato, M., Mizuguchi, T., Hamada, K., Osaka, H., Tohyama, J. et al. De novo mutations in the gene encoding STXBP1 (MUNC18-1) cause early infantile epileptic encephalopathy. Nat. Genet. 40, 782-788 (2008).

27 Minassian, B. A., Lee, J. R., Herbrick, J. A., Huizenga, J., Soder, S., Mungall, A. J. et al. Mutations in a gene encoding a novel protein tyrosine phosphatase cause progressive myoclonus epilepsy. Nat. Genet. 20, 171-174 (1998).

28 Suzuki, T., Delgado-Escueta, A. V., Aguan, K., Alonso, M. E., Shi, J., Hara, Y. et al. Mutations in EFHC1 cause juvenile myoclonic epilepsy. Nat. Genet. 36, 842-849 (2004). 